\title{
Rapid detection of enteric adenovirus and rotavirus: a simple method using polyacrylamide gel electrophoresis
}

\author{
RB MOOSAI, MJ CARTER, CR MADELEY \\ From the Department of Virology, Royal Victoria Infirmary, Newcastle upon Tyne
}

SUMMARY A simple and rapid procedure for identifying adenovirus and rotavirus in stool extracts has been developed. The technique is based on polyacrylamide gel electrophoresis of the virus nucleic acid, but sample preparation is straightforward and does not entail phenol extraction or the use of a radioactive label. Furthermore, processing is not influenced by the amount of specimen obtained and is thus suitable for application as a batch testing method. This approach removes the need for specific antisera, which are not readily available since most of these viruses cannot be grown using routine tissue culture procedures. Trials in this laboratory have indicated that the technique is of comparable sensitivity to electron microscopy.

Several groups of viruses have been implicated in the production of diarrhoeal disease in humans.' Of these, the rotaviruses and adenoviruses are probably the most common, although the involvement of small round viruses, both structured and featureless, should not be underestimated. ${ }^{2}$ Consequently, there is a need to characterise as far as possible the agents responsible for a particular outbreak both for public health reasons and also to gather epidemiological data with which to assess the importance of each type of virus. These viruses were originally discovered in stools by electron microscopy, but they are difficult to isolate in tissue culture and this has led to a search for alternative methods for their detection. Recently, several diagnostic kits have been produced for the detection of rotaviruses. These are based on immunological recognition and are thus limited to the specificity of the antibodies provided. In view of the difficulties experienced in the growth of these viruses in vitro antisera are not readily available and no kit has yet been marketed which can be used to identify enteric adenoviruses. In addition, the application of these procedures is often not straightforward and may be influenced by the amount of specimen or the titre of virus it contains (Moosai et al, unpublished observations). In the absence of sera adequate in quality or quantity, electron microscopy has remained the only method cap-

Accepted for publication 21 August 1984 able of recognising all these virus groups in a single test. The method is simple and rapid, but it is insensitive and confined to viruses which have well defined morphology.

We have recently described the use of polyacrylamide gel electrophoresis (PAGE) in the differentiation of several rotavirus strains, and we have introduced a nomenclature (Rotacode) for the profiles of genomic RNA thus produced. ${ }^{3}$ This approach has now been developed further in order to distinguish the genome of rotavirus (segmented, double stranded RNA) from that of adenovirus (linear, double stranded DNA) and thus provide a diagnostic tool for the identification of these viruses. This technique is simple, convenient, and similar in sensitivity to diagnosis by electron microscopy.

\section{Material and methods}

TREATMENT OF SPECIMENS FOR PAGE AND ELECTRON MICROSCOPY

All stool samples used in this study had been sent to the routine diagnostic laboratory (Royal Victoria Infirmary) and were obtained from children and infants admitted to hospitals in north east England. The stool samples were suspended in Hank's balanced salt solution at a concentration of $10 \%$. This could then be analysed directly by PAGE (see below), but had to be processed further for examination in the electron microscope. Large particles were removed by low speed sedimentation $(2000 \mathrm{rpm}$ in a 
MSE Mistral centrifuge, $4^{\circ} \mathrm{C}$ ) and the virus was concentrated by high speed sedimentation $(45000 \mathrm{rpm}$ for 1 hour in a Beckman Sw 55 rotor at $4^{\circ} \mathrm{C}$ ). Pellets containing virus were resuspended in electron microscopy diluent (distilled water containing $0.1 \%$ bacitracin) and applied to electron microscope grids. The preparations were negatively stained by floating the grids on $2 \%$ potassium phosphotungstate $(\mathrm{pH}$ $7 \cdot 0$ ) for $15 \mathrm{~s}$. The surplus fluid was removed with filter paper and the grids were allowed to dry.

\section{PAGE}

Electrophoresis was performed using the discontinuous gel system described by Laemmli. ${ }^{4}$ Resolving gels $(7.5 \%$ polyacrylamide) were cast between $20 \times 40 \mathrm{~cm}$ glass plates separated by $1.5 \mathrm{~mm}$ spacers, and a stacking gel (3\% polyacrylamide) was then poured on top. Resolving gel mixtures contained: $20 \mathrm{ml}$ of $1.5 \mathrm{M}$ TRIS- $\mathrm{HCl}(\mathrm{pH} 8.8) / 4 \%$ sodium dodecyl sulphate (SDS), $20 \mathrm{ml}$ of $30 \%$ acrylamide $/ 0.8 \%$ bis-acrylamide, $38.7 \mathrm{ml}$ of water. After degassing gels were polymerised with $1.2 \mathrm{ml}$ of $20 \%$ ammonium persulphate and $30 \mu \mathrm{l}$ of $\mathbf{N}, \mathbf{N}, \mathbf{N}^{\prime}, \mathbf{N}^{\prime}$-tetramethylethylenediamine (temed). The stacking gel contained: $6.25 \mathrm{ml}$ of 1 M TRIS$\mathrm{HCl}$ (pH 6.8) $/ 0.8 \%$ SDS, $5 \mathrm{ml}$ of $30 \%$ acrylamide/ $0.8 \%$ bis-acrylamide, $31.3 \mathrm{ml}$ of water. Stacking gels were polymerised with $0.75 \mathrm{ml}$ of $20 \%$ ammonium persulphate and $50 \mu$ lof temed.

Stool suspensions and cell lysates were prepared for electrophoresis in the same way. Each was made $5 \%$ with respect to SDS by the addition of an equal volume of $10 \%$ SDS in distilled water ${ }^{6}$ and incubated for $30 \mathrm{~min}$ at $37^{\circ} \mathrm{C}$ to release nucleic acids and dissociate protein complexes. SDS also acts as a powerful inhibitor of nuclease activity. Then $6.25 \mu \mathrm{l}$ of the treated sample was mixed with an equal volume of sample buffer ( $62 \mathrm{mM}$ TRIS- $\mathrm{HCl} \mathrm{pH} 6 \cdot 8$, $3 \%$ SDS, 5\% 2-mercaptoethanol, and $40 \%$ glycerol) and applied to the gel $(0.5 \mathrm{~cm}$ slot $)$. Gels were electrophoresed for $16 \mathrm{~h}$ at $30 \mathrm{~mA}$ using a "Biorad Protean" cell operated with tap water coolant.

Nucleic acid bands were visualised using the silver staining procedure described by Herring et al, ${ }^{5}$ with slight modifications. Gels were removed from the glass plates and fixed for $60 \mathrm{~min}$ in a solution of $50 \%$ ethanol and $10 \%$ acetic acid. Rehydration was performed in two stages, each of $60 \mathrm{~min}$ duration, by transfer to a solution containing $25 \%$ ethanol, $10 \%$ acetic acid, and thence to $10 \%$ ethanol, $1 \%$ acetic acid. The rehydrated gels were stained in silver nitrate $(0.19 \%$ in water) for a further $60 \mathrm{~min}$, rinsed in distilled water for $10 \mathrm{~min}$, and immersed in a reducing solution $(3 \% \mathrm{NaOH}, 0.37-0.41 \%$ formaldehyde, $0.05 \% \mathrm{NaBH}_{4}$ ) for $10 \mathrm{~min}$ to initiate colour development. Optimum staining was achieved by transferring the gels to sodium carbonate solution $(0.75 \%)$ for $5 \mathrm{~min}$. This procedure stained nucleic acids black in a light brown gel matrix. Wet gels could be stored in fixing solution and kept in the dark until photographed.

DNA molecular weight markers, adenovirus 2, and bacteriophage lambda DNA, were purchased from Sigma Ltd.

\section{GROWTH OF ADENOVIRUS TYPE 2}

Adenovirus type 2 , isolated from a nasopharyngeal secretion, 'was grown at $37^{\circ} \mathrm{C}$ in $\mathrm{HeLa}$ cells under Eagle's minimum essential medium containing $10 \%$ fetal calf serum. Sixteen hours after infection the medium was replaced with Eagle's minimum essential medium containing $2 \%$ serum and incubation was continued. The cells were harvested when the cytopathic effect was maximum (usually about four days after infection) and disrupted by three cycles of freezing and thawing. The resulting cell lysates were clarified by centrifugation ( $1500 \mathrm{rpm}$ for $10 \mathrm{~min})$ in a MSE Mistral centrifuge at $4^{\circ} \mathrm{C}$ and stored frozen. Uninfected $\mathrm{HeLa}$ cells were treated in a similar manner to provide negative controls. All these lysates were processed for electrophoresis exactly as the $10 \%$ stool suspensions described above.

\section{NUCLEASE DIGESTION}

Samples for nuclease digestion were precipitated from the SDS treated preparations with 2.5 volumes of ethanol and collected by centrifugation $(10000 \mathrm{~g}$ for $10 \mathrm{~min}$ ). Nucleic acids were resuspended in 1.5 $\mathrm{M} \mathrm{NaCl}, 0 \cdot 15 \mathrm{M}$ sodium citrate, for treatment with RNAse A (Sigma, $100 \mu \mathrm{g} / \mathrm{ml}$ ) and T1 (Sigma, 100 $\mu \mathrm{g} / \mathrm{ml}$ ), or in $0.1 \mathrm{M}$ TRIS-HCl $(\mathrm{pH} 7.5), 0.1 \mathrm{M} \mathrm{NaCl}$, $15 \mathrm{mM} \mathrm{MgCl}$, for treatment with DNAse 1 (Sigma, $50 \mu \mathrm{g} / \mathrm{ml}$ ). Reaction mixtures were incubated at $37^{\circ} \mathrm{C}$ for $30 \mathrm{~min}$ (RNAse) or $28^{\circ} \mathrm{C}$ for $2 \mathrm{~h}$ (DNAse). Treated samples were mixed with an equal volume of sample buffer and analysed by electrophoresis.

\section{Results}

The protocol described above could be applied routinely to all samples; concentration or dilution according to virus titre was not required. With this procedure, samples containing rotaviruses displayed all the double stranded RNA segments of the virus genome as previously reported. ${ }^{3}$ Stool samples which were known from electron microscopy to contain adenoviruses gave a pattern which was clearly distinguishable from that produced by samples containing rotaviruses, reoviruses, or phage lambda (Fig. 1). Gels run in this manner sometimes showed a diffuse band about halfway down; this was appar- 


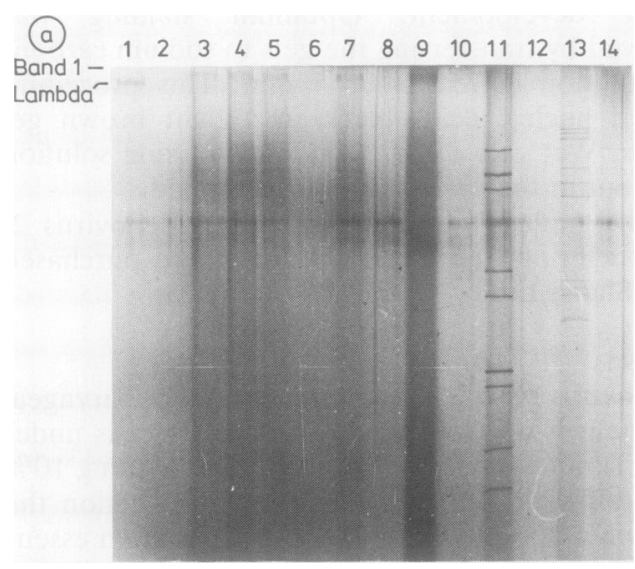

Fig. 1a Analysis of stools containing rotavirus. Stool samples containing rotavirus (tracks 11 and 14), adenovirus (tracks 3-5, 7, 9), or those in which no virus could be detected by electron microscopy $(2,6,8,10,12)$ were electrophoresed in a $7.5 \%$ polyacrylamide gel as described in the text. Nucleic acids prepared from viruses grown in vitro were used as markers: reovirus type 1 (track 13), phage lambda (track 1). The uppermost band (band 1) produced by samples containing adenoviruses is marked. The diffuse band present in all tracks is an artefact of electrophoresis.

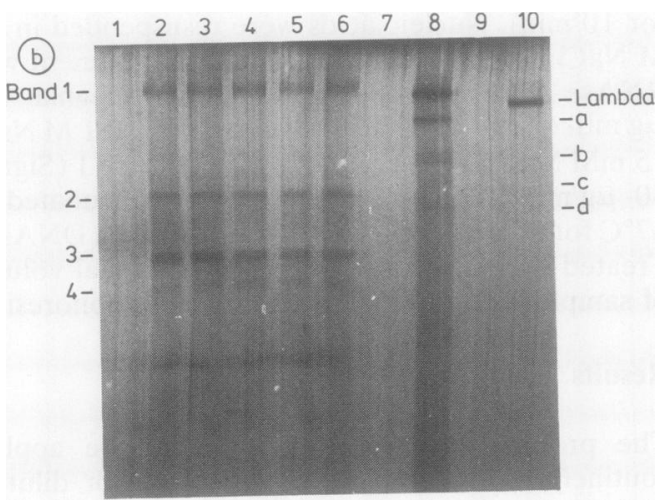

Fig. 1b Comparison of bands produced by stools containing adenovirus (tracks 2-6) with those obtained using adenovirus 2 grown in vitro (track 8 ) and stools in which no virus was detected by electron microscopy (tracks 1 and 7). Small molecular weight species seen in adenovirus positive stools (bands 2-4) and the different species detected in adenovirus preparations grown in vitro $(a-d)$ are indicated. The diffuse band present in all tracks is an artefact of electrophoresis. ent even when no sample was applied and represented an artefact of electrophoresis. Adenovirus positive stool preparations produced a prominent band (band 1 in Fig. 1a, b) near the top of the gel and several others (bands 2-4) of lower molecular weight (Fig. 1b). Virus grown in tissue culture also produced additional bands $(\mathrm{a}-\mathrm{d})$ which were different from those seen in the stool samples (Fig. 1b). The high molecular weight band (band 1) had an almost identical mobility to that observed when adenovirus infected cell lysates or commercially available preparations of adenovirus 2 DNA were analysed on the same gel (Fig. 2). The silver staining procedure is able to detect both protein and nucleic acid, but the high molecular weight band 1, observed in these experiments, was also found to fluoresce when stained with ethidium bromide (not shown), thus confirming that it consisted of nucleic acid. Furthermore, this band was not seen when samples derived from uninfected cells or stools in which no adenovirus had been detected were used (Fig. 1a, b). Consequently, it was likely that the high molecular weight band seen in samples containing adenovirus represented the virus genomic DNA. This was confirmed by testing specimens for DNA content using specific nucleases. The high molecular

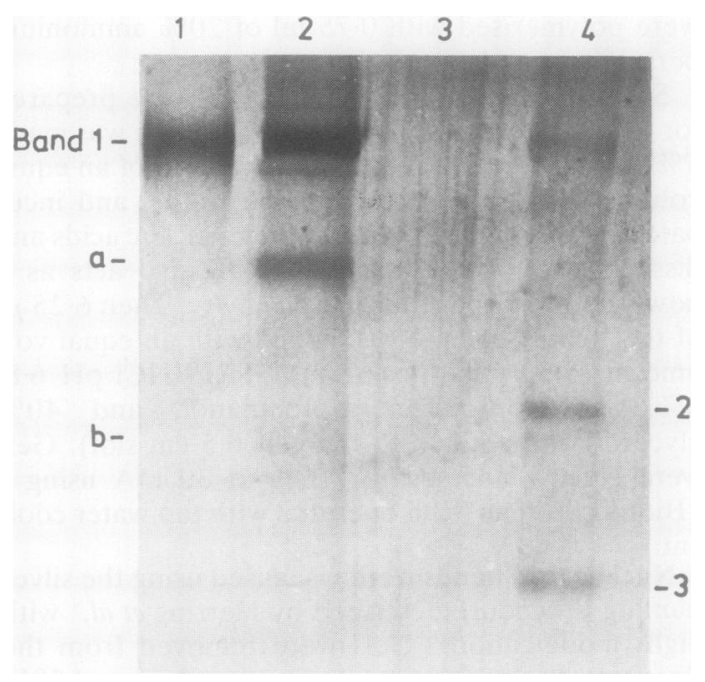

Fig. 2 Identification of adenovirus DNA. Adenovirus DNA samples were electrophoresed on a $7.5 \%$ polyacrylamide gel: track 1, purified adenovirus 2 DNA (Sigma Ltd); track 2, adenovirus 2 grown in tissue culture in this laboratory; track 3, stool sample containing no detectable adenovirus; track 4, stool sample containing enteric adenovirus. Additional DNA bands are visible in both the tissue culture grown virus $(a, b)$ and the enteric virus sample $(2,3)$. 


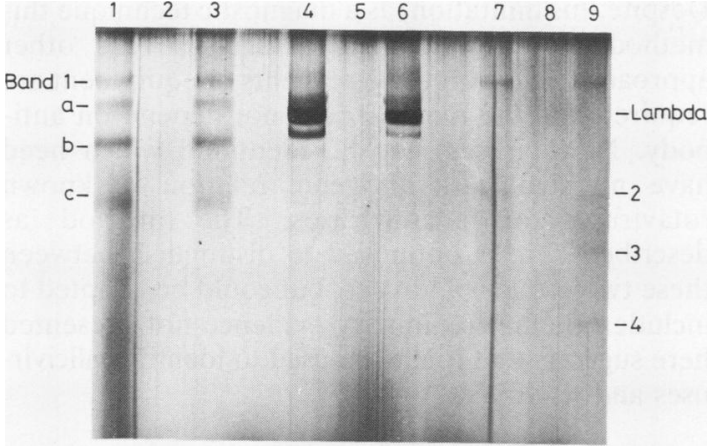

Fig. 3 Nuclease treatment. Effect of nuclease treatment on bands produced by adenovirus or lambda DNA. Tissue culture grown adenovirus, tracks 1-3; bacteriophage lambda DNA, tracks 4-6; enteric adenovirus, tracks 7-9. Samples applied to tracks 2, 5, and 8 have been treated with DNAse 1. Samples applied to tracks 3, 6, and 9 have been treated with RNAses A and T1. Additional bands present in the tissue culture grown adenovirus $(a-c)$ and the enteric virus (2-4) are also marked.

weight band was resistant to ribonucleases $A$ and $T 1$ but sensitive to deoxyribonuclease. A purified preparation of bacteriophage lambda DNA (MW $31 \times$ $10^{6}$ ) was used as a positive control in these experiments and showed an identical pattern of sensitivity to digestion by these enzymes (Fig. 3). We therefore concluded that band 1 consisted of DNA which displayed electrophoretic migration properties almost identical to those of adenovirus genomic DNA and which could be used as a diagnostic feature for the detection of this virus group. The lower molecular weight bands were also stained by ethidium bromide and digested by DNAse (Fig. 3), thus showing that they were also DNA. Although found only in association with band 1 DNA, they varied widely in amount, and thus were not considered suitable for use in virus identification. Consequently, these bands have not been investigated further. The lambda DNA migrated with an anomalously high mobility in this SDS-PAGE system and formed a band below the adenovirus DNA, which is known to be smaller (MW $\left.25 \times 10^{6}\right)$. This was found to be an artefact of PAGE. Using the same samples the adenovirus DNA migrated more rapidly than the lambda DNA in $0.5 \%$ agarose gels, as expected.

The efficiency of diagnosis by PAGE was compared with that of diagnosis using the electron microscope by conducting a "blind" trial. Sixty stool samples known to be either positive (44) or negative (16) for adenovirus by electron microscopy were coded by one worker and then tested by PAGE. In all cases samples found to contain adenoviruses by microscopy were correctly identified by PAGE. In one case a sample which was negative for virus by electron microscopy was positive for adenovirus by PAGE. This sample was then re-examined in the microscope and adenovirus particles were found, although in lower numbers than in the other samples. Consequently, both methods agreed in all cases examined.

\section{Discussion}

We have described a method which we believe could provide a rapid and reliable means of detecting adenoviruses and rotaviruses in stool suspensions. PAGE has been applied to this problem previously, but has suffered from the necessity to prepare pure nucleic acid which could then be radiolabelled in vitro. This was a cumbersome technique which required expensive reagents. ${ }^{6}$ The use of the silver staining procedure has removed this limitation and permitted the use of simple nucleic acid extraction procedures. Deproteinisation is not required; after combination with SDS proteins acquire a large negative charge and presumably migrate through the gel more rapidly than the nucleic acids. Stool samples are also rich in protease ${ }^{7}$ so that any high molecular weight proteins are probably degraded in these specimens. Protease activity would also help inactivate nucleases in the stools. These factors may explain the surprising effectiveness of this approach and the clarity of the results.

The finding of DNA species of lower molecular weight is as yet unexplained. These molecules, found in stool samples containing adenovirus, were of similar size, although they often varied in amount. They have never been detected in specimens containing rotavirus or in samples negative for both viruses. Consequently, they do not seem likely to cause any confusion in the identification of stool viruses. The stools used in these experiments in which no virus could be detected (negative stools) were obtained from patients with gastrointestinal symptoms. This makes it unlikely that such additional bands have arisen as a byproduct of the illness. Their association with enteric adenovirus specimens suggests that they may represent incomplete adenovirus or adeno-associated virus genomes. The extra bands noted in preparations of adenovirus grown in tissue culture may also represent incomplete genomes. These species of lower molecular weight were not considered in the diagnosis of adenovirus. Band 1 DNA was sufficient for this purpose and the method was of equal sensitivity to electron microscopy when applied to clinical specimens. This technique should be suitable for routine specimen testing since samples are all treated in the same manner. The virus specific DNA bands were 
identified by the use of specific nucleases, but this is not necessary on a routine basis. Provided marker nucleic acids are used, we have found that the identification of adenovirus DNA may be made on the basis of comigration alone. Each gel should therefore include a sample of commercially obtained adenovirus DNA as well as stool samples known to contain adenovirus and rotavirus as markers. Any artefactual effect on the migration of adenovirus DNA in PAGE is fully reproducible, and it is not therefore necessary to use agarose gels as well. Furthermore, the silver staining method is not applicable to agarose gels and it is more complicated to ensure sufficient material is loaded for clear detection by ethidium bromide staining.

The findings presented here suggest that the PAGE test could be used instead of electron microscopy for routine diagnosis. However, at least in the early stages, it is anticipated that both techniques would be used in parallel. Later on, only samples showing unusual PAGE patterns-for example, the atypical rotaviruses-need be rechecked in the electron microscope.

In the case of the rotaviruses, different virus strains may be identified by the particular pattern the segmented genome produces on electrophoresis (electrophoretype). This may be useful in assessing the epidemiological importance of individual strains. A similar analysis is not possible for adenoviruses because their genome is not segmented and differences in mobility between strains are small or absent. Consequently, the further investigation of adenovirus epidemiology will depend on the identification of individual virus strains, and we are currently extending these experiments to permit such identification using restriction enzymes.
Despite this limitation, as a diagnostic technique this method has major advantages over other approaches: no expensive reagents or equipment are required and the method does not depend on antibody. New viruses may be identified which need have no significant antigenic relation to known rotaviruses or adenoviruses. The method as described here is optimised to distinguish between these two groups of viruses, but could be adapted to include others. Preliminary evidence not presented here suggests that it may be used to identify caliciviruses and reoviruses.

This work was supported by a grant from the Medical Research Council. We thank R Alcock, K English, and D Lee for technical assistance.

\section{References}

' Holmes IH. Viral gastroenteritis. Prog Med Virol 1979; 25: 1-36.

${ }^{2}$ Madeley CR. Viruses in the stool. J Clin Pathol 1979;32:1-10.

${ }^{3}$ Moosai RB, Alcock R, Madeley,CR. A cryptogram for recording rotavirus strains: the rotacode. J Hyg (Camb) (in press).

${ }^{4}$ Laemmli UK. Cleavage of structural proteins during the assembly of bacteriophage T4. Nature 1970;227:680-5.

${ }^{5}$ Herring AJ, Inglis NF, Ojeh CK, Snodgrass DR, Menzies JD. Rapid diagnosis of rotavirus infection by direct detection of viral nucleic acid in silver stained polyacrylamide gels. $J$ Clin Microbiol 1982; 16:473-7.

- Clarke IN, McCrae MA. A rapid and sensitive method for analysing the genome profiles of field isolates of rotavirus. $J$ Virol Meth 1981;2:203-9.

${ }^{7}$ Moosai RB, Gardner PS, Almeida JD, Greenaway MA. A simple immunofluourescent technique for the detection of human rotavirus. J Med Virol 1979;3:189-94.

Requests for reprints to: Dr MJ Carter, Department of Virology, The Royal Victoria Infirmary, Queen Victoria Road, Newcastle upon Tyne NE1 4LP, England. 